(c) 2010 IEEE. Personal use of this material is permitted. Permission from IEEE must be obtained for all other uses, in any current or future media, including reprinting/republishing this material for advertising or promotional purposes, creating new collective works, for resale or redistribution to servers or lists, or reuse of any copyrighted component of this work in other works. 


\section{SMART CAMP: Environmental Sustainability Through Intelligent Automation Technologies}

\author{
Markus M. Kohn \\ Faculty of Digital Media \\ Hochschule Furtwangen \\ University \\ Furtwangen, Germany \\ m.kohn@hs-furtwangen.de
}

\author{
Elizabeth Chang \\ Digital Ecosystems and \\ Business Intelligence \\ Institiute \\ CBS, Curtin University \\ of Technology \\ Perth, Australia \\ Elizabeth.Chang@cbs.curtin \\ .edu.au
}

\author{
Achim P. Karduck \\ Faculty of Digital Media \\ and Computer Science \\ Hochschule Furtwangen \\ University \\ Furtwangen, Germany \\ karduck@hs-furtwangen.de
}

\author{
Alex Talevski \\ Digital Ecosystems and \\ Business Intelligence \\ Institute \\ CBS, Curtin University \\ of Technology \\ Perth, Australia \\ A.Talevski@curtin.edu.au
}

\begin{abstract}
A "Smart Camp" solution is a new approach to provide a mining community consisting of multiple households in a remote area in Western Australia with a intelligent wireless network and automated information system. This system intends to automate, control and monitor numerous household devices; supplies the tenants with multi-media services and aims to reduce energy consumption as well as improving the workers habitat. This paper theorizes the big picture with considerations on: how to set up, integrate and create a cost-effective "smart" wireless home automation network solution. It attempts to improve employee quality of life while simultaneously reducing energy consumption and the related emissions. Therefore, the latest Wireless Technologies in the field of domotics will be discussed and the importance of environmental sustainability and energy awareness will be outlined.
\end{abstract}

Keywords-component; Smart Camp; Environmental Sustainability; Home Automation; Smart Home; Remote Areas; Remote Camps; Intelligent Information Systems; Domotics; ZigBee Wireless Networks; Convergent Media; Media-enriched Services; Smart Appliance; Value-added Service;

\section{INTRODUCTION}

In the last few years, network enabled technologies like WLAN, Ethernet or Bluetooth have seen significant adoption and have made their ways into residential homes. On the other hand, the adoption of home automation networks in peoples homes has been quite limited. As [1] points out, research on domotics is broadly handed down by the academic arena and only few industrial research programs exist. Although the field of home automation has existed for decades with X10 as the first industry standard for interconnecting electronic devices, it hasn't made a big impact on residential living and larger communities and in industrial camps. The reasons for this are manifold. Recent systems were based on powerline or wired communication technologies. These systems are intrusive, complex, expensive and mostly only fit new buildings and complexes. New wireless network enabled technologies (e.g. ZigBee and Z-Wave) seem to solve these problems as they take the advantage of being flexible, easier to implement, interoperable and cost effective.
These wireless network systems also seem to offer a new approach to energy conservation and environmental sustainability. They can include devices to control and monitor the energy consumption.

The term sustainability or sustainable development was defined by the 1987 Brundtland Report of the World Commission on Environment and Development [20] as "development that meets the needs of the present without compromising the ability of future generations to meet their own needs." This is not limited to, but implies that it is necessary to increase the efficency of the energy consumption to reduce the environmental impact. Environmental sustainability is one of the most serious and compelling challenges faced by scientists nowadays. Although governments have began to act, a community wide approach is necessary with a larger fraction of individuals that are committed to reduce energy consumption.

Presently the Government of Westeren Australia [9] is implementing a plan for an Energy Smart Program, which aims to reduce the state's environmental impact and energy consumption. Part of this program is the promotion and administration of a "Renewable Remote Power Generation Program" which intends to improve renewable energy technology in remote areas of Western Australia, as well as the promotion of awareness of house energy rating tools and energy-efficient options for residential homeowners. This example shows not only the importance, but also the great demand on sophisticated energy saving techniques, such as wireless home automation networks, for households in remote areas within Australia and other countries.

In recent years, there has been a new term introduced in the fields of Computer Science and domotics - "Smart Home" to describe new technologies which aim to reach existing goals. [17] quotes the following definition on a "Smart Home". A "Smart Home" is "(...) a dwelling incorporating a communications network, that connects the key electrical appliance and services, and allows them to be remotely controlled, monitored or accessed." This definition reveals, that 
there have been no new starting point for thoughts, yet. The new smart technologies offer a bigger potential than this.

We define the word "Smart Camp" in a enhanced way. A Smart Camp is a integrated "smart" system of different kinds of network technologies and devices including media-enriched value-added services for multiple households in a camp, to serve the need for comfort and increase the quality of life of the inhabitants, while enabling environmental sustainability due to energy conservation and awareness.

Therefore building up a Smart Camp system means to consider both; technology issues as well as human factors.

This project focuses on new challenges to optimise rural camp management and operation, and employee quality of life while reducing power consumption and emissions. The goal of the project is to investigate sustainable Smart Camp operation through the use of state-of-the-art intelligent automation and information technologies. The project strives to contribute to the spread of wireless domotic technologies as well as it intends to encourage environmental awareness and saving.

In this paper, we aim to indentify a novel stand alone lowcost and flexible ZigBee based Solution and demonstrate that the Smart Camp initiative is a sustainable philosophy.

\section{REQUIREMENTS}

In this section, we define the requirements for the project. For this work, the requirements for the proposed system fit into four main categories. The first category defines the general requirements. The next two categories show the requirements for the stakeholders, the tenants and the camp operators. The fourth category shows the technical requirements.

\section{A. Environmental requirements :}

- Environment effects system: Harsh, remote environment demands a system which fits to that need.

- System effects environment: Energy saving and environmental conservation due to reduce the total diesel consumption and $\mathrm{CO}^{2}$ emission as a consequence and encourage environmental sustainability.

\section{B. Requirements from tenants point of view:}

- Temperature control; the tenants should be able to control their room temperature individually.

- Access control; the tenants should be able to access their room through a standardized solution. Security must be guaranteed to the tenant.

- Multi-media / entertainment; the tenants should be supplied with a convergent (all-in-one) media solution.

- Energy usage control; the tenants should be able to monitor their energy usage at any time.

- $\quad$ Ease of Use; tenants should experience the system as usable. They should be able to operate the system without deep technical skills and with their own technical devices (e.g. smart phones) if desired.

\section{Requirements from camp operators point of view:}

- Occupant compliance; to run the system efficiently, the system should be highly accepted by the tenants.

- Energy usage and cost reduction; the system has to contribute energy saving. Diesel usage should be reduced by thirty per cent.

- Cost effectiveness; the suggested solution should return on investment after 2 years.

- Camp Site Energy Control (realtime); to measure energy and cost saving goals, the whole power consumption of the camp site should be monitored in realtime.

- Camp Site Monitoring; enhanced security due to the realtime monitoring of occupancy status for each Single Person Quarters (SPQ) unit.

\section{Basic technical requirements:}

From a technical point of view the proposed system must offer the following functionalities:

- Media-enriched services: including TV and additionally Pay-TV (via smart card). Internet access via Television Screen or additional PC. VoIP Solution, which enables communication functionalities. Additonaly tenants should be able to connect and use the Internet connection with their mobile devices. All the services must be accessible for every tenant.

- Heating Ventilating and Air Conditioning (HVAC) control. To save energy, the air conditioning unit has to automatically reduce or switch off the cooling power when the SPQ unit is unattended. The temperature should be at the same level when the tenant returns.

- A wireless door access system has to be integrated to every SPQ unit. The environmental conditions like dust and sand demand a locking system without physical user interface elements (e.g. buttons, keys or card readers).

- A low rate wireless personal area network technology with a low power consuption, that links data of the system components together and enables them to communicate with each other.

- A computer with a low-power CPU, that implements the multi-media and network technologies and acts as a central coordinator for each SPQ unit sub network. This computer should be easy to install and easy to maintain by the camp coordinators.

- A control centre that provides the camp operators with the gathered data of all SPQ units to monitor energy usage in realtime. This control centre can rather be installed on-site or remotely managed from any other place in the world.

- Extensibility for devices and sensors. 


\section{ARCHITECTURE}

To demonstrate the feasibility and effectiveness of the proposed system, a Smart Camp will be set up for the proof of concept. As mentioned above, the system has to meet certain requirements. Our approach is based on different system components that will be integrated into a Smart Camp.

\section{A. System Components}

- (1) Smart Camp Management Unit (SCMU) - This application is what is described above as a control centre. It is a scalable application which remotly controls one or more camps and it is administrated by a security camp operator. The SMCU is considered to be the backbone of the entire system. It gathers the data from all of the Smart Camp Box' to monitor all activities of the camp including power consumption and status of occupancy of each unit.

- (2) SPQ unit - The Single Person Quarter unit denotes the accomodation for the workers of the mining camp. The main energy consumer in each SPQ is considered to be the air condition $(\mathrm{A} / \mathrm{C})$, which causes approx. $80 \%$ to $90 \%$ of the power consumption.

- (3) Smart Camp Box - The SCB is the central controller for the SPQ unit. It has an integrated ZigBee Gateway and communicates to all other devices in the household. Therefore, it sends and receives messages from and to all the other ZigBee devices. It also inherits the media-enriched services: The SCB provides Internet and VoIP-Service to the tenants via Ethernet cabling. The Ethernet connection is also used to communicate to the SCMU.

- (4) In-Home Display - The IHD will be located in a prominent position to make it an interactive feature of the household. The IHD shows the current power usage and the costs for the SPQ unit. The aim of the display is to motivate the occupants to save energy by giving incentives for acting responsible.

- (5) HVAC Control - The HVAC control is also connected to the SCB via ZigBee. The HVAC control, for example, is connected to the thermostat or the IRInterface of an $\mathrm{A} / \mathrm{C}$. It retrieves and forwards status data to the $\mathrm{A} / \mathrm{C}$ system like temperature and fan speed. The SCB collects the data and automatically decreases the temperature or turns the $\mathrm{A} / \mathrm{C}$ off, when the SPQ unit is unattended for longer than 20 minutes.

- (6) Door Lock - The proposed Wireless Door Lock will be using RFID-Technology for the locking system, as well as ZigBee or RS-232 cabeling to communicate with the SCB. The SCB keeps track of all access activities. It'll notice if the SPQ is occupied and forward a request to the HVAC unit to turn on the $\mathrm{A} / \mathrm{C}$. Malfunctions will be forwarded from the Door Lock to the SCB; and from the SCB to the SCMU, where a camp operator keeps track of all requests and alerts.

- (7) Additional Devices - After installing a system, it should be possible to integrate other devices.
- (8) Ethernet Connection - Each SCB is connected to the SCMU via Ethernet. The Ethernet connections are already provided by the camps.

- (9) ZigBee Wireless Network - The Zigbee Network is the communication channel for all power consuming devices; especially for the A/C. Each device will communicate through a Zigbee Chip with the coordinator.

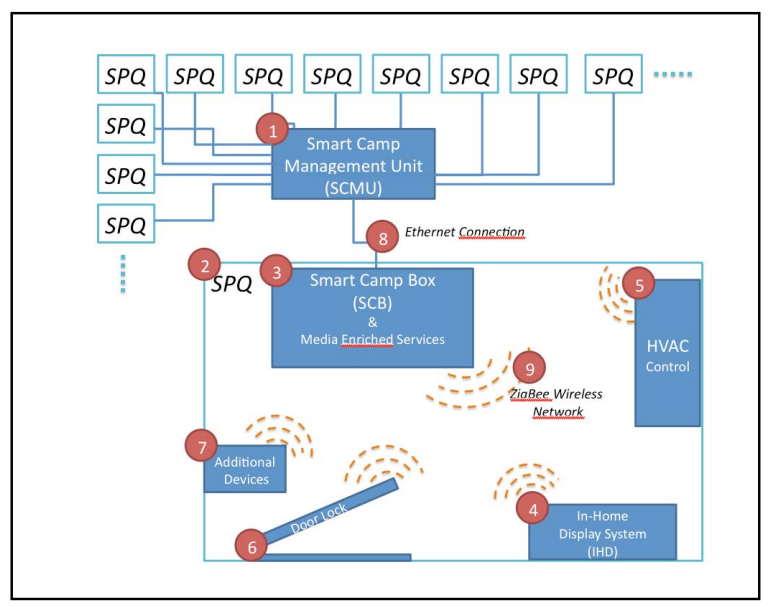

Figure 1. Smart Camp Architecture

\section{B. Functionality of the proposed solution}

The above architecture can also be summarized by the provided functionalities (figure 2). (1) to (6) show the functionalities that are inherit by the SCB. It includes (1) (Pay)TV, (2) VoIP, (3) Web Browser, (4) A/C regulation, (5) RFID access control and the extensibility for other devices. (7) The SCMU has the abiltiy to remotly monitor and control the Smart Camp.

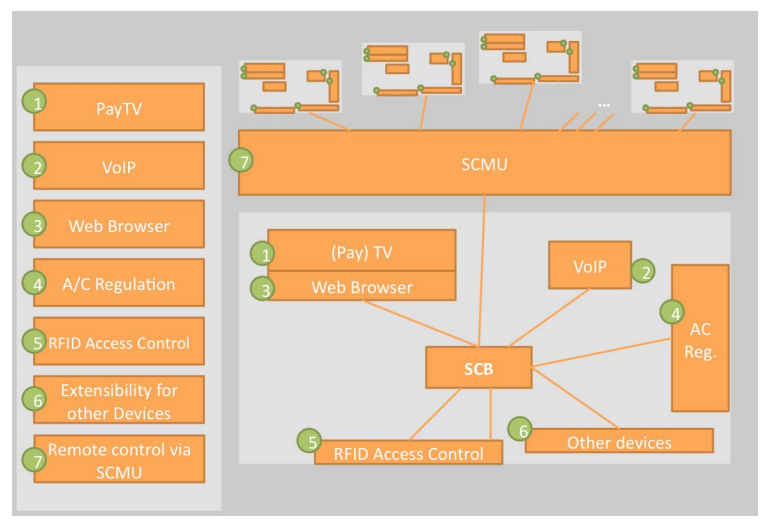

Figure 2. Functionality of the proposed solution 


\section{COMPONENTS AND INFRASTRUCTURE IMPLEMENTATION}

\section{A. The IEEE802.15.4 Standard}

As ZigBee is based on the underlying radio standard IEEE802.15.4 for wireless communication (established by the Institute for Electronic Engineers, IEEE) we will describe this structure and difficulties in general. The standard is specified for communication in a point-to-point or a point-to-multi configuration with sleeping and security functions as integral parts of the standard [6]. It was developed with a lower rate, than IEEE802.11 or IEEE802.16 and a simpler connection with the intention of enabling battery powered applications. All of these applications involve a central coordinator. The coordinator acts as a data collector with multiple remote nodes connecting back to this central host. For our purpose, the central coordinator will be integrated into the SCB and will be responsible for creating and maintaining the network.

Two PHY layers are defined by the IEEE 802.15.4 which represent three license-free frequency bands that include sixteen channels at $2.4 \mathrm{GHz}$ in Australia [14].

For the set up of a personal area network (PAN), the IEEE802.15.4 standard allows the arrangement of two different types of network topologies. The star and the Peer-to-peer topology.

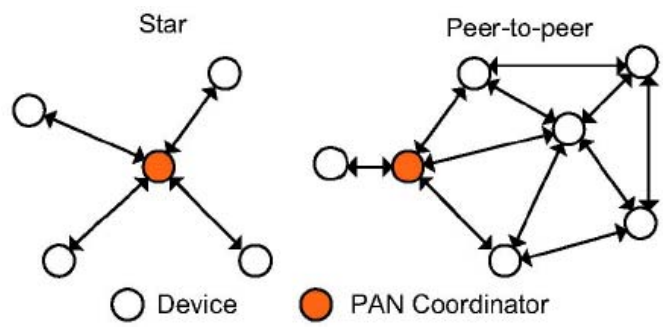

Figure 3. PAN Topologies [13]

A single central controller also named the PAN coordinator performs the communication between the devices in the star topology. The PAN coordinator, therefore, is in charge of managing all the star PAN functionality [13].

Network devices can communicate to others on the network in its range using a Peer-to-peer topology. In this case, the PAN coordinator acts like a root of this network. The use of Peer-topeer topology allows the implementation of more complex network formations such as self-healing and self-configuring networks. The address of the PAN is commonly shared between all of the devices in a PAN.

For our purposes, star as well as peer-to-peer topology can be taken into consideration. Star topology offers a straight forward approach, with the SCB as central PAN coordinator, deploying and logging the data to all other network devices.

On the other hand, the peer-to-peer topology offers some security aspects like the self-healing of the network. The peerto-peer topology can also cause some problems, as the devices from different vendors are not interoperable because of different application profiles. Mesh topology demands more power from each device, which means batteries have to be replaced more often. Both PAN topology settings will be tested, when the Smart Camp prototype is set up, to evaluate the best outcome [13].

The Physical (PHY) layer of IEEE802.15.4 provides four main functions:

- $\quad$ Spreading and de-spreading

- Modulation and demodulation of the signal

- Capability of measuring the power of the received signal

- Adjusting the connection quality

Direct Sequence Spread Spectrum (DSSS) is used as spreading technique in IEEE802.15.4. As modulation type in the $2.4 \mathrm{GHz}$ band O-QPS with a $32 \mathrm{PN}$-code length and a RF bandwidth of $2 \mathrm{MHz}$ is used. BPSK modulation with a $15 \mathrm{PN}-$ code length is used in the sub-1 GHz bands and it operates in the $600 \mathrm{KHz}$ RF bandwidth [14]. The network of the Smart Camp will operate in the $2.4 \mathrm{GHz}$ band.

The MAC Layer offers seven main functions [19]:

- Control to the access of the communication channel

- Flow control through acknowledgements and retransmissions

- Responsibility for data validation and synchronisation

- Packaging of data into frames before transmission

- Unpacking frames and checking errors

- $\quad$ Providing service to the upper layers

- Monitoring of the channels through the use of CSMA/CA algorithm

The MAC sub layer provides access to the physical channel for all types of transfer and assures the reliable transfer of the frames. Therefore, it uses a simple light weight protocol, that is based on CSMA and it includes the transmitting of beacon frames, synchronization and provides a reliable transmission mechanism [13].

\section{B. The ZigBee Protocol}

The ZigBee protocol is a product of the ZigBee Alliance The Zigbee Alliance is an industry group that supervises the development of the standard. It focuses on the standardization of Low Rate Wireless Personal Area Networks (LR-WPAN) technology for applications in the industrial settings, building automation, and consumer markets as well as on the certification of products and brands compliant with the ZigBee standard. By defining the upper layers of the protocol stack, the Alliance is aiming to create secure, multi-hop LR-WPAN networks and interoperable applications [18].

The ZigBee protocol uses the IEEE802.15.4 standard and adds an additional network layer to enable routing and networking functionality for the system. Signal bandwidth, frequency and modulation techniques are defined by the PHY and MAC layer of IEEE802.15.4. 
There are two generic types of 802.15.4 LR-WPAN nodes upon which ZigBee devices are based:

- Reduced Function Devices (RFD) - As RFD only operate as reduced complexity nodes with relatively limited memory, processing, and power capabilities, they can only serve as End Devices in a network and cannot perform the more complex roles of Router or Coordinator [18], [19].

- Full Function Devices (FFD) - The FFD devices have the resources to perform more complex task such as Coordinator or Router but can also be an End Device in a network.

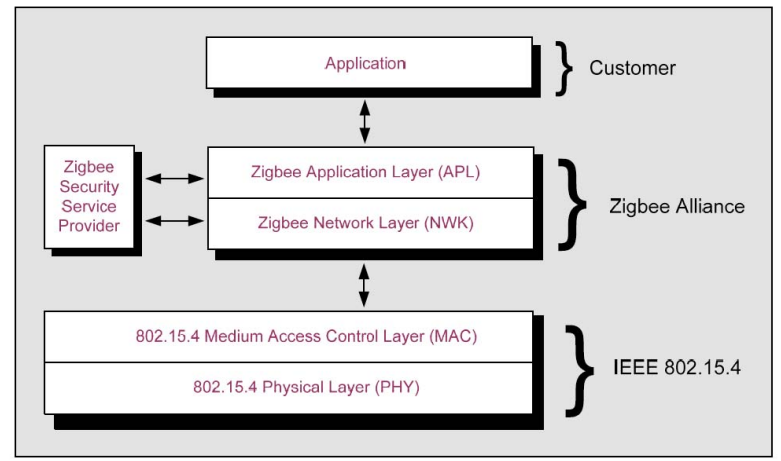

Figure 4. Simplyfied ZigBee Architecture [18]

The central concept of the ZigBee Application Layer is the Application Profile. Profiles are the key to communicating between devices on a ZigBee network. The Application Profiles define the devices, messages, and processing actions that comprise of an application running among ZigBee End Devices in a given environment in order to ensure compatibility and interoperable functionality between them. There is an Application Layer frame that is 16-bits in length called the Profile Identifier field, which specifies the profile [18]. Because every ZigBee Device vendor can specify its own profile, this causes problems of interoperability between different devices. To assure that all the devices will interoperate, only compatible items with common profiles must be integrated.

The Zigbee Application Layer also contains the ZigBee application framework. It is the environment in which ZigBee objects are hosted by the devices. As much as 240 different application objects can be defined, each identified by an endpoint address from 1 to 240. Two additional endpoints are already defined: endpoint 0 is reserved for the data interface to the Zigbee Device Object (ZDO) and endpoint 255 for the data interface function to broadcast data to all application objects [19].

\section{Recent Research on the ZigBee Protocol}

[5], [6] focus on the implementation of ZigBee Wireless Networks, interoperability between ZigBee devices and how to integrate ZigBee networks into others e.g. for remote monitoring. As critical building automation applications, like surveillance and door lock systems, requires end-to-end Quality of Service, [5] proposes a system architecture which is capable of dealing with the stringent and often conflicting requirements for wireless building automation by combining heterogeneous technologies into a suitable networking solution. [6] suggests a ZigBee-based Radio-Frequency Identification (RFID) Network, which is used for real time monitoring and locating objects.

Considering how the technology can interact seamlessly with consumer attendance and behavior, [7] proposes an indoor positioning system via ZigBee devices. Another approach [16] presents a solution, using an array of pyroelectric infrared sensors (PIR sensors), that are able to detect residents. Systems like these are costly, not reliable and hard to implement for huge communities, but should be considered as an option for further optimizations on the Smart Camp.

Other approaches, like [8], address human factors by considering human-system interaction, quality of life and value added services, when it comes to implementing home automation environments and wireless networks. [15] states that, although smart home appliances are capable of delivering new functions and services, there is still a huge gap between user habits and the services offered. The reason given for this is, that organizations still focus on specific fields for service and appliance design rather than to plot the smart home as a whole.

In order to enable value-added services for the users, [15] proposes two major objectives: (1) aims on content sharing among devices, to meet the demand of entertaining and information appliances. (2) focuses on home automation to control the status of appliances and for better operation modes and quality of life improvements.

\section{Energy Measurement and Awareness}

There are many different approaches on how to monitor, measure and display the power consumption of a household to its inhabitants. [10] shows that the approach of In-Home Displays (IHD) can achieve consumption savings of 5-15 per cent if they are part of a wider plan to save household resources. Based on evidence from existing Australian trials, they also found, that electricity savings of up to four per cent are likely through an IHD, without any other implementations. As [10] points out, the powerfulness of the IHD device " $(. .$. lies in its situated nature within the habitat of the household, and its ability to 'script' or 'moralise' certain behaviors". [11] states that it is useful to households to have a feedback information, which is specific to them and allows them to control their energy use more effectively. Therefore, mature key-drivers for the design of a IHD are user-friendliness, the display of instantaneous usage, expenditure and historic feedback as a minimum and also the potential for displaying information on microgeneration, tariffs and carbon emissions.

\section{Challenges}

In this section we describe, what we consider as the six main difficulties of the project. 


\section{A. Environment}

For our purpose, the system is intended to be implemented as an real world project in a remote camp in the Western Australian Outback. Multiple factors like the high temperature, sand and dust, as well as power provided by diesel generators cause harsh environmental conditions, that could affect the reliability and the life cycle of the electronic devices. Devices must be build robust enough to assure Quality of Service and low maintenance.

\section{B. Implementation}

As we aim to implement the Smart Camp system in a real world environment, it has to be a system which is easy to set up, install and implement to avoid high costs.

\section{Technology Integration}

As pointed out earlier, the integration of different kinds of technologies is one of the key issues. Additionally, not only domotic devices will be integrated, but also additional mediaenriched services will be implemented. As this is a novel way, we choose a bottom-up approach to merge different kinds of technologies.

\section{Cost Effectiveness}

The Smart Camp also has to be cost-effective. Not only the price for all system components should be reasonable, but also the costs of maintenance should be very low. Therefore the system has to be reliable, scalable and easy to maintain.

\section{E. Energy Consumption}

At present, there is no fully validated information available on how much energy a SPQ unit is consuming under a given set of conditions. As there are no established methods or protocols for minimizing SPQ energy usage, a methodology will be established, which will make it possible to measure and rate the energy consumption and reduction.

The goal is to reduce the overall energy consumption of a SPQ unit by $30 \%$. After present investigations, the $A / C$ is considered to be the highest consumer of power in the SPQ. Therefore, we aim to throttle down the energy of $\mathrm{A} / \mathrm{Cs}$, when SPQ units are unattended. The aim is to implement a HVAC control solution, which is coupled to the door lock, an attendance sensor system (e.g. motion sensors) and a employee roster. The idea is to link the behavior of the $\mathrm{A} / \mathrm{C}$ according to the presence of the inhabitants. The $\mathrm{A} / \mathrm{C}$ should automatically be throttled down to a lower cooling intensity when the SPQ unit is unattended and returned to the desired cooling level at least half an hour before the occupants return to the unit.

\section{F. Ease of Use and Adoption}

The Smart Camp should be easy to use for the tenants. After a first introduction, the occupants should be able to manage the system without any deeper knowledge of the technology. It is important, that handling and navigation of the $\mathrm{SCB}$, as well as the IHD and thermostats are intuitive to their users and cannot be easily manipulated.
The system has to be adopted by the tenants of the SPQs. The usage of IHD and RFID access systems will effect everyday life of the inhabitants. To give incentives for the usage of the Smart Camp, media-enriched services will be provided. [12] is focusing on this part of the project.

\section{CONCLUSIONS}

The paper presented a proposal for an intelligent system prototype for sustainable Smart Camp management. The big picture of the design and implementation of a Wireless ZigBee Network Solution was presented.

A Smart Camp prototype will be built to verify the performance and reliability of our proposal. After achieving this, testing the performance in real camp sites and under real world conditions is desired.

\section{REFERENCES}

[1] K. Gill, S. H. Yang, F. Yao and X. Lu, "A ZigBee-based home automation system," Consumer Electronics, IEEE Transactions on, vol. 55, pp. 422-430, May 2009.

[2] A. Sikora, "Wireless sensor networks," Wireless Congress Munich, 2007.

[3] C. Reinisch, W. Kasnter, G. Neugschwandtner and W. Granzer, "Wireless technologies in home and building automation" in Industrial Informatics, 2007 5th IEEE International Conference on, vol. I, 23-27 Jun, 2007, pp. 93-98.

[4] M. Osipov, "Home automation with ZigBee," in Next Generation Teletraffic and Wired/Wireless Advanced Networking, vol. 5174/2008, 23-27 Jun, 2008, pp. 263-270.

[5] W. Vandenberghe, B. Latr'e, F. De Greve, P. De Mil, S. Van den Berghe, K. Lamont, I. Moerman, M. Mertens, J. Avonts, C. Blondia and G. Impens, "A system architecture for wireless building automation," Original Source: Proceedings of the 15th IST Mobile \& Wireless Communications Summit 2006.

[6] M. Sumi, A. E. Soujeri, R. Rahim, A. I. Harikrishnan, "Design of a ZigBee-based rfid network for industry applications," SIN '09: Proceedings of the 2nd international conference on Security of information and networks, Oct 2009, pp. 111-116.

[7] H. Pensas, H. Raula, and J. Vanhala, "Energy efficient sensor network with service discovery for smart home environments," Sensor Technologies and Applications, 2009. SENSORCOMM '09. Third International Conference on, pp. 399-404, June 2009.

[8] T. Yamazaki, "Beyond the smart home" in Hybrid Information Technology, 2006. ICHIT '06. International Conference on, vol. II, 0911 Nov, 2007, pp. 350-355.

[9] Government of Western Australia, Office of Energy, "Annual report 2008/09," Office of Energy , 2009, pp. 22-25, pp. 58-60, http://www.energy.wa.gov.au/cproot/1572/14919/Office\%20of\%20Ener gy\%202008\%2009\%20Annual\%20Report_FINAL_Lo\%20Res.pdf.

[10] Y. Strengers, "Smart metering demand management programs: challenging the comfort and cleaniness habitus of households,"OZCHI '08: Proceedings of the 20th Australasian Conference on ComputerHuman Interaction: Designing for Habitus and Habitat, vol. 287, 9-16 Nov, 2007, pp. 9-16.

[11] S. Darby, "The effectiveness of feedback on energy consumption," A Review for DEFRA of the literature on metering, billing and direct display, Environmental Change Institute, University of Oxford, Oxford Eng land, April 2006.

[12] L. J. Oslislo, A. Talevski, A. P. Karduck "Smart camp - benefits of media and smart service convergence," unpublished.

[13] C. Bolzani, C. Montagnoli and M. Netto, "Domotics over ieee 802.15.4 a spread spectrum home automation application," 2006 IEEE Ninth 
International Symposium on Spread Spectrum Techniques and Applications, 2006, pp. 395-400.

[14] V. Goyal, V. Mittal and M. Aggarwal, "Technique for making zigbee power efficient," International Conference on Advaces in Computing, Communication and Control (ICAC3'09), 2009, pp. 92-95.

[15] C. Chun-Yu, T. Yu-Ping, L. Shu-Chen and L. Cheng-Ting, "Implementing the design of smart home and achieving energy conservation," Industrial Informatics, 2009. INDIN 2009. 7th IEEE International Conference on, 23-26 June 2009, pp. 273 - 276.

[16] K. Nam Ha, K. Chang Lee and S. Lee, "Development of pir sensor based indoor location detection sysem for smart home," SICE-ICASE International Joint Conference 2006, Oct. 18-21, 2006 in Bexco, Busan, Korea,
[17] J. Li, L. Da-You and Y. Bo, "Smart home research," Proceedings of the Third International Conference on Machine Learning and Cybernetics, Shanghai, 2004, Aug. 26-29, pp. 659-663.

[18] K. Masica, "Recommended practices guide for securing zigbee wireless networks in control system environments,"

[19] ZigBee Alliance, "Zigbee specification, ZigBee Document 053474r17," 2007, ZigBee Standards Organization, http://www.zigbee.org/ZigBee SpecificationDownloadRequest/tabid/311/Default.asp

[20] World Commission on Environment and Development. Our Common Future; Oxford University Press: Oxford, UK, 1987.

[21] United Nations General Assembly (2005). 2005 World Summit OutcomeResolution. 